\title{
Episodic long-term memory in post-infectious SARS-CoV-2 patients
}

\author{
Edoardo Nicolò Aiello ${ }^{1}$ (D) Elena Fiabane ${ }^{2} \cdot$ Marina Rita Manera $^{3} \cdot$ Alice Radici $^{4} \cdot$ Federica Grossi $^{3}$. \\ Marcella Ottonello ${ }^{2} \cdot$ Claudio Vassallo ${ }^{2} \cdot$ Debora Pain $^{4} \cdot$ Caterina Pistarini $^{5}$
}

Received: 6 October 2021 / Accepted: 13 November 2021 / Published online: 17 November 2021

(c) Fondazione Società Italiana di Neurologia 2021

\begin{abstract}
Background Episodic long-term memory (LTM) difficulties/deficits are frequent in COVID-19-recovered patients and negatively impact on prognosis and outcome. However, little is known about their semiology and prevalence, also being still debated whether they arise from primary amnesic features or are secondary to dysexecutive/inattentive processes and disease-related/premorbid status. Hence, this study aimed at (1) assessing LTM functioning in post-infectious SARS-CoV-2 patients by accounting for premorbid and disease-related confounders and (2) exploring its cognitive etiology.

Methods Measures of global cognition (Mini-Mental State Examination (MMSE) and Montreal Cognitive Assessment (MoCA)) and LTM (Babcock Memory Test (BMT)) of fifty-four COVID-19-recovered patients were retrospectively collected. Patients were subdivided into those being already at risk or not for cognitive decline (RCD +; RCD -). Cognitive measures were converted into equivalent scores (ESs).

Results LTM sub-clinical/clinical deficits $(\mathrm{ESs}=0 / 1)$ were mildly-to-moderately prevalent in both RCD + (MoCA-Memory, 31.8\%; BMT, 31.8\%) and RCD - (MoCA-Memory, 28.6\%; BMT, 39.3\%) patients. MMSE and MoCA total scores, but not the MoCA-Attention subtest, were associated with the BMT. RCD + asymptomatic patients performed better on the BMT $(p=.033)$ than those requiring $\mathrm{O}_{2}$ therapy (but not ventilation).

Discussion COVID-19-recovered individuals might show LTM deficits of both primary and secondary etiology and should be thus screened for them, especially those having suffered mid-to-moderate COVID-19 and those already being at risk for cognitive decline. Both I- and II-level measures of verbal LTM can be adopted, although the former might be more sensitive.
\end{abstract}

Keywords SARS-CoV-2 $\cdot$ COVID-19 $\cdot$ Memory $\cdot$ Cognitive assessment $\cdot$ Attention

Edoardo Nicolò Aiello

e.aiello5@campus.unimib.it

Elena Fiabane

elenamaria.fiabane@icsmaugeri.it

Marina Rita Manera

marina.manera@icsmaugeri.it

Alice Radici

alice.radici@icsmaugeri.it

Federica Grossi

federica.grossi@icsmaugeri.it

Marcella Ottonello

marcella.ottonello@icsmaugeri.it

Claudio Vassallo

claudio.vassallo@icsmaugeri.it

Debora Pain

debora.pain@icsmaugeri.it
Caterina Pistarini

caterina.pistarini@icsmaugeri.it

1 PhD Program in Neuroscience, School of Medicine and Surgery, University of Milano-Bicocca, Monza, Italy

2 Istituti Clinici Scientifici Maugeri, Department of Physical and Rehabilitation Medicine of Genova Nervi Institute, Genova, Italy

3 Istituti Clinici Scientifici Maugeri, IRCCS, Psychology Unit of Pavia Institute, Pavia, Italy

4 Istituti Clinici Scientifici Maugeri, IRCCS, Neurorehabilitation Department of Milano Institute, Milano, Italy

5 Istituti Clinici Scientifici Maugeri, IRCCS, Department of Neurorehabilitation of Pavia Institute, Pavia, Italy 


\section{Introduction}

Cognitive sequelae of COVID-19 within the dysexecutiveinattentive and amnesic spectrum have been attributed to neurotropic properties of SARS-CoV-2 and featured neuroinflammatory processes [5], as well as to iatrogenic confounders (e.g., steroidal treatments) [4] and premorbid risk factors for cognitive impairment [1]. To screen for cognitive deficits in this population has been stressed as relevant due to their adverse impact on rehabilitative and ecological outcomes [5].

Subjective episodic long-term memory (LTM) difficulties are frequently reported by COVID-19-recovered patients [8] also yielding at psychometric testing [5] and neuroradiological examinations of LTM-related structures [6]. However, little is known about the semiology and prevalence of post-COVID-19 LTM difficulties, being still debated whether such deficits arise from primary amnesic features due to medial temporal dysfunctions [7] or are secondary to non-instrumental processes of a prefrontal etiology [10]. Further knowledge on this can help practitioners with the cognitive diagnostics in this population by selecting appropriate psychometric tools $[1,7]$.

Thereupon, this study aimed at (1) assessing LTM functioning in a clinic-based cohort of post-infectious SARS$\mathrm{CoV}-2$ patients by also accounting for premorbid and diseaserelated confounders and (2) exploring its cognitive etiology.

\section{Methods}

\section{Materials}

Data from fifty-four COVID-19-recovered patients referred to ICS Maugeri, IRCCS Pavia (Northern Italy) have been retrospectively collected (Table 1).

According to Aiello et al. [1], patients were subdivided into those already at risk or not for cognitive decline (RCD+; RCD-) based on remote, recent, and COVID-19-related medical records.

Patients underwent global cognitive screening via the MiniMental State Examination (MMSE) [3] and the Montreal Cognitive Assessment (MoCA) [2] as well as a II-level evaluation of verbal episodic LTM via the Babcock Memory Test (BMT) [9].

\section{Statistics}

Analyses were conducted separately for RCD+ and RCD- patients.

As data adequately converged to a normal distribution (skewness and kurtosis values $<|1|$ and $|3|$, respectively), linear model analyses were run to test associations/predictions.
MMSE, MoCA, and BMT scores were adjusted for anagraphic-demographic confounders and converted into equivalent scores (ESs) in order to draw clinical judgments $[2,3,9]$.

Agreements between ES-standardized clinical judgments were performed via weighted Cohen's $k$.

SPSS 27 (IBM Corp., 2020) was adopted to analyze data; significance level $(\alpha=.05)$ was Bonferroni-corrected for multiple comparisons when adequate.

\section{Results}

$\mathrm{RCD}+$ and $\mathrm{RCD}-$ groups were balanced as to the majority of background and clinical variables, except for sex, disease severity, and ICU admission rates; moreover, RCD+ patients reported significantly lower MMSE and MoCA total adjusted scores (ASs) when compared to the RCD- group (see Table 1). LTM deficits as detected by the MoCA-Memory and the BMT were mildly-to-moderately prevalent in both groups (see Table 1).

LTM sub-clinical and clinical deficits (defined as $\mathrm{ESs}=1$, i.e., "borderline," and 0, i.e., "impaired," respectively) were detected in $31.8 \%$ of RCD- and $28.6 \%$ of RCD+ patients by the MoCA-Memory, whereas in $31.8 \%$ of RCD- and $39.3 \%$ of RCD+ patients by the BMT. However, substantial disagreements in classifying patients with clinical/sub-clinical deficits ( $E S=0$ and $E S=1$, respectively) yielded when comparing the MoCA-Memory subtest and the BMT in both groups (RCD-, $k=-.26, p=.228 ; \mathrm{RCD}+, k=.14, p=.463)$ with the BMT trending to classify RCD+ patients that performed "normally" at the MoCA-Memory as sub-clinically/ clinically impaired $(N=7)$ (Table 2$)$.

When exploring the association between raw scores at the BMT and remaining cognitive measures, the former proved to be related to the MMSE in both groups (RCD-, $r(22)=.44 ; p=.039 ; \mathrm{RCD}+, r(28)=.49 ; p=.0098)$, whereas with the MoCA in RCD+ patients only $(r(28)=.52$; $p=.005)$. In both groups, neither MoCA-Attention nor MoCA-Memory raw scores were significantly associated with BMT raw scores ( $|.03| \leq r \leq 1.37 \mid ; p \geq .051)$.

No significant association was found between BMT, MoCA-Attention, and MoCA-Memory ASs and either disease duration $\left(|.04| \leq r_{s} \leq 1.281 ; p \geq .202\right)$, time from onset $\left(|.006| \leq r_{s} \leq 1.22 \mid ; p \geq .27\right)$, ICU admission $(|.02| \leq t \leq|2.07|$; $p \geq .052)$ or steroidal treatment ( $1.08|\leq t \leq| 1.83 \mid ; p \geq .084)$. Although disease severity did not affect BMT, MoCAAttention, and MoCA-Memory ASs scores in the RCD - group $(.5 \leq F \leq 3.43 ; p \geq .054)$, BMT adjusted scores yielded to be significantly influenced by disease severity in RCD+ patients $(F(3,24)=3.92 ; p=.021)$, with asymptomatic patients $(M=11.5 ; S D=3.85)$ performing better 
Table 1 Patients' background, clinical, and cognitive measures

\begin{tabular}{|c|c|c|c|}
\hline & RCD- & $\mathrm{RCD}+$ & $p \dagger$ \\
\hline$N$ & 22 & 28 & - \\
\hline Age (years) & $66.45 \pm 9.91(47-85)$ & $69.25 \pm 10.81(46-85)$ & .351 \\
\hline Sex (male/female) & $19 / 3$ & $14 / 14$ & $.007^{*}$ \\
\hline Education (years) & $10.91 \pm 3.09(3-22)$ & $11.93 \pm 3.44(5-18)$ & .332 \\
\hline Disease duration (days) & $39.95 \pm 23.43(12-99)$ & $40.96 \pm 29.14(2-113)$ & .9 \\
\hline Time from onset (days) & $70.48 \pm 34.49(26-173)$ & $73.25 \pm 44.23(7-241)$ & .813 \\
\hline Severity & & & $.029^{*}$ \\
\hline Asymptomatic & - & $14.3 \%$ & - \\
\hline Mildly symptomatic & $4.5 \%$ & $10.7 \%$ & - \\
\hline Mild-to-moderate & $13.6 \%$ & $28.6 \%$ & - \\
\hline Moderate-to-severe & $81.8 \%$ & $46.4 \%$ & - \\
\hline ICU & $68.2 \%$ & $35.7 \%$ & $.045^{*}$ \\
\hline Steroids & $45.5 \%$ & $57.1 \%$ & .209 \\
\hline \multicolumn{4}{|l|}{ MMSE } \\
\hline Adjusted scores & $27.76 \pm 2.04(22.11-30)$ & $26.09 \pm 2.66(19-30)$ & $.019^{*}$ \\
\hline Below cut-off $\%$ & $4.5 \%$ & $28.6 \%$ & - \\
\hline \multicolumn{4}{|l|}{ MoCA-Total } \\
\hline Adjusted scores & $22.1 \pm 2.55(15.65-26.35)$ & $19.97 \pm 3.88(13.85-26.17)$ & $.024 *$ \\
\hline Below cut-off $\%$ & $9.1 \%$ & $35.7 \%$ & - \\
\hline \multicolumn{4}{|l|}{ MoCA-Attention } \\
\hline Adjusted scores & $4.75 \pm 1.22(1.82-6)$ & $4.64 \pm 1.53(1.84-6)$ & .796 \\
\hline Below cut-off $\%$ & $13.6 \%$ & $28.6 \%$ & - \\
\hline \multicolumn{4}{|l|}{ MoCA-Memory } \\
\hline Adjusted scores & $1.22 \pm 1.25(-.24-4.18)$ & $1.41 \pm 1.35(-.24-4.62)$ & .617 \\
\hline Below cut-off $\%$ & $13.6 \%$ & $14.3 \%$ & - \\
\hline \multicolumn{4}{|l|}{ Babcock Memory Test } \\
\hline Adjusted scores & $8.73 \pm 2.51(3.3-13.4)$ & $8.37 \pm 3.82(0-14.3)$ & .686 \\
\hline Below cut-off $\%$ & $2 \%$ & $17.9 \%$ & - \\
\hline
\end{tabular}

$\mathrm{RCD}+$, patients at risk for cognitive deficits; $\mathrm{RCD}-$, patients not at risk for cognitive deficits; MMSE Mini-Mental State Examination, MoCA Montreal Cognitive Assessment, ICU intensive care unit ${ }^{\dagger} p$-values refer to either $\chi^{2}$ (categorical measures) or $t / F$ statistics (continuous measures)

*significant at $\alpha=.05$
Table 2 Classification of patients reporting $\mathrm{ESs}=0 / 1$ at the MoCAMemory subtest $v s$. the BMT

\begin{tabular}{|c|c|c|c|c|}
\hline & & \multicolumn{2}{|c|}{ MoCA-Memory } & \\
\hline & BMT & $\mathrm{ES} \leq 1$ & $\mathbf{E S} \geq \mathbf{2}$ & Total \\
\hline \multirow[t]{3}{*}{ RCD- } & $E S \leq 1$ & 1 & 6 & 7 \\
\hline & $E S \geq 2$ & 6 & 9 & 15 \\
\hline & Total & 7 & 15 & 22 \\
\hline \multirow[t]{3}{*}{ RCD+ } & $\mathrm{ES} \leq 1$ & 4 & 7 & 11 \\
\hline & $E S \geq 2$ & 4 & 13 & 17 \\
\hline & Total & 8 & 20 & 28 \\
\hline
\end{tabular}

ES equivalent score, MoCA Montreal Cognitive Assessment, BMT Babcock Memory Test; RCD -, patients not at risk for cognitive deficits; RCD +, patients at risk for cognitive deficits. ESs are to be interpreted as follows: $\mathrm{ES}=0 \rightarrow$ "impaired"; $\mathrm{ES}=1 \rightarrow$ "borderline"; $\mathrm{ES} \geq 2 \rightarrow$ "normal". Diagonal cells show agreements; extra-diagonal cells show disagreements
( $p=.033$ ) than those requiring $\mathrm{O}_{2}$ therapy but not ventilation (mild-to-moderate; $M=5.31 ; S D=3.2$ ).

\section{Discussion}

Episodic LTM sub-clinical/clinical deficits proved to be mildly-to-moderately prevalent in post-infectious SARSCoV-2 patients-the higher rate being found in those already at risk for cognitive decline [5]. LTM deficits could be detected by both I- and II-level measures of verbal LTM, although the latter proved to be slightly more sensitive than the former, especially with respect to $\mathrm{RCD}+$ patients.

As to the cognitive etiology of LTM deficits, I-level measures of attention (MoCA-Attention) did not prove to be associated with II-level LTM measures. By contrast, the latter were related to measures of global cognition (MMSE, 
MoCA), suggesting that LTM deficit in these populations may be partially accounted for by a general decrease in cognitive efficiency. Therefore, although primary amnesic features could not be ruled out [7], the present findings suggest that LTM deficits are, to an extent, secondary to impairments of non-instrumental functions. This would find endorsement in prefrontal circuitries possibly being one of the main targets of SARS-CoV-2 neurotropism [10].

Finally, steroidal treatments, although posited to iatrogenically affect medial-temporal structures [4], were not found to be associated with LTM deficits. By contrast, selective LTM deficits yielded in RCD+ patients requiring $\mathrm{O}_{2}$ therapy (but not ventilation).

As to limitations, only one II-level, verbal LTM measure was adopted: future studies should thereupon focus on tests assessing different facets of LTM (e.g., prospective), also through visuo-spatial materials. Furthermore, no neuroradiological support of these cognitive findings was provided, thus making further anatomo-clinical investigations on LTM deficits in COVID-19 patients necessary.

In conclusion, COVID-19-recovered individuals might show LTM deficits of diverse etiology, especially those having suffered mid-to-moderate COVID-19 and those already at risk for cognitive decline. To screen for such deficits, both I-level and domain-specific measures of verbal LTM can be adopted, although the former might be more sensitive.

\section{Declarations}

Patients provided informed consent. This study received approval by the local Ethics Committee (I.D.: 2470, 8 September 2020).

Conflict of interests The authors declare no competing interests.

\section{References}

1. Aiello EN, Fiabane E, Manera MR, Radici A, Grossi F, Ottonello M, Pain D, Pistarini C (2021) Screening for cognitive sequelae of SARS-CoV-2 infection: a comparison between the MiniMental State Examination (MMSE) and the Montreal Cognitive Assessment (MoCA). Neurol Sci 1-4. https://doi.org/10.1007/ s10072-021-05630-3

2. Aiello EN, Gramegna C, Esposito A, Gazzaniga V, Zago S, Difonzo T, Maddaluno O, Appollonio I, Bolognini N (2021b) The Montreal Cognitive Assessment (MoCA): updated norms and psychometric insights into adaptive testing from healthy individuals in Northern Italy. Aging Clin Exp Res 1-8

3. Carpinelli Mazzi M, Iavarone A, Russo G, Musella C, Milan G, D’Anna F, Garofalo E, Chieffi S, Sannino M, Illario M, De Luca V (2020) Mini-Mental State Examination: new normative values on subjects in Southern Italy. Aging Clin Exp Res 32:699-702

4. Chen F, Hao L, Zhu S, Yang X, Shi W, Zheng K, Wang T, Chen H (2021) Potential adverse effects of dexamethasone therapy on COVID-19 patients: review and recommendations. Infect Dis Ther 10:1907-1931

5. Daroische R, Hemminghyth MS, Eilertsen TH, Breitve MH, Chwiszczuk LJ (2021) Cognitive impairment after COVID-19 a review on objective test data. Front Neurol 12:1238

6. Guedj E, Million M, Dudouet P, Tissot-Dupont H, Bregeon F, Cammilleri S, Raoult D (2021) 18 F-FDG brain PET hypometabolism in post-SARS-CoV-2 infection: substrate for persistent/ delayed disorders? Eur J Nucl Med Mol Imaging 48:592-595

7. Ritchie K, Chan D, Watermeyer T (2020) The cognitive consequences of the COVID-19 epidemic: collateral damage? Brain Commun 2:069

8. Søraas A, Bø R, Kalleberg KT, Støer NC, Ellingjord-Dale M, Landrø NI (2021) Self-reported memory problems 8 months after COVID-19 Infection. JAMA Netw Open 4:e2118717-e2118717

9. Spinnler H, Tognoni G (1987) Standardizzazione e taratura italiana di test neuropsicologici. Neurol Sci 8:1-120

10. Toniolo S, Di Lorenzo F, Scarioni M, Frederiksen KS, Nobili F (2021) Is the frontal lobe the primary target of SARS-CoV-2?. J Alzheimer's Dis 81:75-81

Publisher's note Springer Nature remains neutral with regard to jurisdictional claims in published maps and institutional affiliations. 